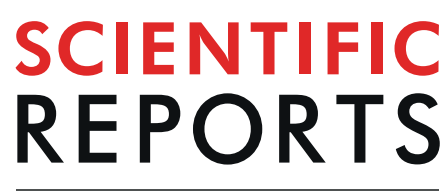

natureresearch

\title{
WNT gene polymorphisms and predisposition to apical periodontitis
}

\author{
Letícia Chaves de Souza $\mathbb{1}^{1,2}$, Franco Cavalla $2,3,4$, Lorena Maili5,6, Gustavo P. Garlet $\mathbb{1}^{4}$, \\ Alexandre R. Vieira $\mathbb{1}^{7}$, Renato M. Silva ${ }^{1,2}$ \& Ariadne Letra $\mathbb{1}^{2,6,8^{*}}$
}

Single nucleotide polymorphisms (SNPs) in WNT genes may impact gene/protein function and contribute to individual predisposition to apical periodontitis (AP). Here, we investigated the association of SNPs in/nearby WNT3, WNT3A, WNT5A, WNT8A, WNT9B and WNT11 genes with AP using a case-control dataset. Cases were defined as individuals with deep caries and AP ( $n=188)$; controls had deep caries and no AP $(n=230)$. Genotyping was performed using Taqman chemistry in real time PCR. Data analyses was performed using Fisher Exact tests assuming a Bonferroni correction threshold value of 0.005 . Single-SNP association analysis revealed a trend for association with WNT3 rs9890413 genotypes $(P=0.009)$ under a dominant model and allelic association for WNT3A rs 1745420 $(P=0.009)$. Haplotypes involving WNT3-WNT9B-WNT3A alleles were also significantly associated with AP $(P \leq 0.003)$. Luciferase reporter assays showed higher transcriptional activity (1.4-fold) with the alternate $G$ allele in rs1745420. Expression of WNT3, WNT3A and WNT5A in AP tissues was significantly higher than in control tissues, and inversely correlated with the expression of SERPINB1, COLIA1 and TIMP1 $(P<0.05)$. Our results suggest that WNT genes have a role in modulating AP and polymorphisms in these genes may increase susceptibility to AP.

Apical periodontitis (AP) represents a local immune response to the progression of microorganisms from an infected root canal space to the periapical area that results in bone resorption characterized by a periapical radiolucency ${ }^{1}$.

AP has heterogeneous etiology, where different bacterial combinations can result in similar clinical outcomes ${ }^{2}$. Furthermore, the endodontic microbiota varies between individuals and each individual presents a unique bacterial profile, regarding the type of species present and their abundance ${ }^{2}$. Endodontic pathogens present in teeth diagnosed with pulp necrosis and AP have been associated with the expression of mediators related to soft tissue and bone destruction in AP, such as matrix metalloproteinases (MMPs)-2 and -9, receptor activator of NFKB (RANK), RANK ligand (RANKL) and osteoprotegerin (OPG). These findings suggest a dynamic process between the microbial aggression and host responses during AP development ${ }^{3}$. In accordance with these previous studies, single nucleotide polymorphisms (SNPs) in disease-relevant genes, mostly belonging to immune-response related pathways, have recently been reported in association with AP predisposition ${ }^{4-6}$.

The Wnt/ $\beta$-catenin signaling pathway, also known as the canonical Wnt pathway, controls cell differentiation, growth, proliferation, survival, and immune cell function and has been linked to several skeletal diseases and cancers ${ }^{7}$. Wnt interactions with Frizzled (Fzd) and low-density lipoprotein receptor related protein 5/6 (LRP5/6) receptors result in cytoplasmic accumulation of $\beta$-catenin and its translocation into the nucleus, where it binds to $\mathrm{T}$-cell factor/lymphoid enhancer factor (TCF/LEF) to regulate the transcription of target genes ${ }^{7}$. In bone, activation of Wnt/ $\beta$-catenin signaling increases bone mass by regulating stem cell renewal, stimulating pre-osteoblast

${ }^{1}$ Department of Endodontics, University of Texas Health Science Center School of Dentistry, Houston, 77054, USA. ${ }^{2}$ Center for Craniofacial Research, University of Texas Health Science Center School of Dentistry, Houston, 77054, USA. ${ }^{3}$ Department of Conservative Dentistry, School of Dentistry, University of Chile, Santiago, 7520355, Chile. ${ }^{4}$ Department of Biological Sciences, Bauru School of Dentistry, University of São Paulo, Bauru, 17012, Brazil. ${ }^{5}$ Department of Pediatrics, University of Texas Health Science Center at Houston McGovern Medical School, Houston, 77030, USA. ${ }^{6}$ Pediatric Research Center, University of Texas Health Science Center at Houston McGovern Medical School, Houston, 77030, USA. 'Department of Oral Biology, University of Pittsburgh, Pittsburgh, 15229, USA. ${ }^{8}$ Department of Diagnostic and Biomedical Sciences, University of Texas Health Science Center School of Dentistry, Houston, 77054, USA. *email: Ariadne.M.Letra@uth.tmc.edu 


\begin{tabular}{|c|c|c|c|c|c|}
\hline Chr. & Gene & SNP ID & Base Position $^{\mathrm{a}}$ & SNP location ${ }^{\mathrm{a}}$ & Alleles $^{\mathrm{a}, \mathrm{b}}$ \\
\hline \multirow{4}{*}{1} & \multirow{4}{*}{ WNT3A } & rs708111 & 228003664 & Upstream & G/A \\
\hline & & rs3094912 & 228022114 & Intron 1 & $\mathrm{~T} / \mathrm{C} / \mathrm{A}$ \\
\hline & & rs752107 & 228059650 & $3^{\prime}$ prime UTR & T/C \\
\hline & & rs1745420 & 228064031 & Downstream & G/A/C \\
\hline 3 & WNT5A & rs566926 & 55486750 & Intron 6 & T/A/G \\
\hline 5 & WNT8A & rs 2040862 & 138084300 & Intron 3 & $\mathrm{C} / \mathrm{T}$ \\
\hline 11 & WNT11 & rs1533767 & 76194756 & Exon 6 & G/A \\
\hline \multirow{3}{*}{17} & \multirow{3}{*}{ WNT3 } & rs9890413 & 46824083 & Upstream & G/A \\
\hline & & rs199498 & 46788237 & Intron 1 & $\mathrm{~T} / \mathrm{C} / \mathrm{G}$ \\
\hline & & rs111769 & 46794621 & Intron 1 & $\mathrm{C} / \mathrm{T}$ \\
\hline 17 & WNT9B & rs2165846 & 46864000 & Intron 2 & A/G \\
\hline
\end{tabular}

Table 1. Details of WNT genes investigated. Chr., chromosome; SNP, single nucleotide polymorphism. aAccording to Ensembl GRCh38.p12 assembly (June 21, 2019). ${ }^{\mathrm{b}}$ Ancestral allele in bold reported on the forward strand.

replication and osteoblastogenesis as well as inhibiting osteoblast apoptosis ${ }^{8}$. Previous studies using animal models of AP reported a significant increase in the expression of Wnt family member 5 A (WNT5A) in AP tissues which correlated with the severity of inflammation ${ }^{9}$. Furthermore, additional studies showed the upregulation of WNT gene expression during periapical lesion development, and suggested that the AP-induced inflammation inhibits osteoblast differentiation via modulation of Wnt/ $\beta$-catenin signaling pathway ${ }^{8,10,11}$.

In this study, we investigated whether SNPs in WNT genes may contribute to AP risk. We also assessed the function of associated SNPs and mRNA expression analysis of WNT genes in AP tissues.

\section{Results}

Genetic association analysis. We used a case-control candidate gene approach to investigate the association of SNPs in/nearby WNT genes with AP (Table 1). Our study population consisted of 188 cases with AP (individuals with deep caries and AP $>3 \mathrm{~mm}$ in diameter) and 230 controls (individuals with deep caries but no AP). Individuals were recruited at the UTHealth School of Dentistry at Houston and at the University of Pittsburgh School of Dental Medicine Dental Registry and DNA Repository under IRB-approved protocols and informed consent. Diagnosis of AP was obtained by an endodontist based on clinical and radiographic examinations of each individual. Genomic DNA samples were used as templates for genotyping.

Eleven SNPs in/nearby WNT3, WNT3A, WNT5A, WNT8A, WNT9B and WNT11 genes were selected for genotyping based on published reports, locations within their respective genes, potential functional consequences (i.e., located in regulatory regions such as promoter, $3^{\prime}$ UTR, exon, or exon/intron boundaries), or if considered tag-SNPs as surrogates for the linkage disequilibrium blocks surrounding the contributor gene ${ }^{12}$ (Table 1).

Association analysis was performed in the combined datasets, and also stratified by recruitment site, considering a Bonferroni correction alpha value of 0.005. A first-pass analysis showed that two SNPs (rs566926 and rs2040862) were in deviation from Hardy-Weinberg equilibrium (HWE) which were excluded from further analysis.

Under a strict Bonferroni correction criteria $(\alpha=0.005)$, analysis of the combined dataset showed a trend for positive association for WNT3 (rs9890413) in individuals with deep caries and AP, both under allelic and dominant genotype models $(\mathrm{P}=0.007)$. This association appeared to be driven by the association values obtained for the Pittsburgh dataset $(\mathrm{P}=0.009)$. Suggestive association was also found between $W N T 3 A$ rs 1745420 alleles and a deep caries with AP phenotype in the Houston dataset $(\mathrm{P}=0.009)$. Additional nominal associations $(\mathrm{P}<0.05)$ were found for other WNT genes (Table 2 and Supplementary Tables S1-S3).

Significant haplotype associations were also observed and included the individually associated SNPs (Table 3 and Supplementary Table S4). In the combined dataset, the strongest association was observed for the combination of WNT3 rs111769 and rs9890413 alleles (CG haplotype, $\mathrm{P}=0.0002$ ), followed by WNT3 rs111769/ rs9890413 and WNT9B rs2165846 alleles (CGG haplotype, $\mathrm{P}=0.0003)$, WNT3 rs199498/rs111769/rs9890413 alleles (TCG haplotype, $\mathrm{P}=0.002$ ), WNT3 rs199498/rs111769/rs9890413 and WNT9B rs2165846 alleles (TCGG haplotype, $\mathrm{P}=0.002$ ), and WNT3 rs9890413/WNT9B rs2165846 alleles (GG haplotype, $\mathrm{P}=0.004$ ) (Table 3 and Supplementary Table S4). In the Pittsburgh dataset, the strongest association was observed for the combination of WNT3 rs111769/rs9890413 alleles (CG haplotype, $\mathrm{P}=0.00008$ ), followed by WNT3 rs111769/rs9890413 and WNT9B rs2165846 alleles (CGG haplotype, $\mathrm{P}=0.0002)$, WNT3 rs199498/rs111769/rs9890413 alleles (TCG haplotype, $\mathrm{P}=0.0005$ ), and WNT3 rs199498/rs111769/rs9890413 and WNT9B rs2165846 (TCGG haplotype, $\mathrm{P}=0.0009$ ) (Table 3 and Supplementary Table S4). In the Houston dataset, association was observed for the combination of WNT3A rs752107 and rs 1745420 alleles (CC haplotype, $\mathrm{P}=0.003$ ) (Table 3 and Supplementary Table S4).

Functional analysis of associated SNPs. We used the FuncPred tool on the SNPinfo web server (https:// snpinfo.niehs.nih.gov/snpinfo/snpfunc.html) and TargetScan (http://www.targetscan.org) for in silico prediction of SNV function for the associated WNT3 rs9890413 and WNT3A rs1745420. These SNVs are located upstream and downstream of their respective genes and might have potential regulatory effects on gene and/or encoded 


\begin{tabular}{|c|c|c|c|c|c|c|c|c|c|c|}
\hline Gene & SNP Id ${ }^{a}$ & Studied population & $\begin{array}{l}\text { MAF } \\
\text { CEU }^{\mathrm{a}, \mathrm{b}}\end{array}$ & $\begin{array}{l}\text { MAF } \\
\text { (cases) }\end{array}$ & \begin{tabular}{|l|} 
MAF \\
(control)
\end{tabular} & Test & Alleles & 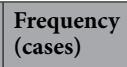 & $\begin{array}{l}\begin{array}{l}\text { Frequency } \\
\text { (controls) }\end{array} \\
\end{array}$ & Pvalue \\
\hline \multirow{5}{*}{ WNT3 } & \multirow{5}{*}{ rs9890413 } & \multirow{2}{*}{ Pittsburgh } & $0.36(\mathrm{G})$ & 0.39 & 0.28 & Allelic & G/A & $77 / 119$ & $75 / 193$ & 0.01 \\
\hline & & & & & & Recessive & GG/GA+AA & $14 / 84$ & $12 / 122$ & 0.20 \\
\hline & & \multirow{3}{*}{$\begin{array}{l}\text { Combined } \\
\text { (Pittsburgh + Houston) }\end{array}$} & & & & Genotypic & GG/GA/AA & $22 / 85 / 70$ & $17 / 80 / 111$ & 0.02 \\
\hline & & & $0.36(\mathrm{G})$ & 0.36 & 0.27 & Allelic & G/A & $129 / 225$ & $114 / 302$ & 0.007 \\
\hline & & & & & & Recessive & GG/GA+AA & $22 / 155$ & $17 / 191$ & 0.17 \\
\hline \multirow{4}{*}{ WNT3A } & \multirow{4}{*}{ rs1745420 } & \multirow{4}{*}{ Houston } & & & & Genotypic & $\mathrm{CC} / \mathrm{CG} / \mathrm{GG}$ & $10 / 26 / 42$ & $16 / 33 / 25$ & 0.04 \\
\hline & & & $0.22(\mathrm{C})$ & 0.29 & 0.44 & Allelic & $\mathrm{C} / \mathrm{G}$ & $46 / 110$ & $65 / 83$ & 0.009 \\
\hline & & & & & & Dominant & $\mathrm{CC}+\mathrm{CG} / \mathrm{GG}$ & $36 / 42$ & $49 / 25$ & 0.01 \\
\hline & & & & & & Recessive & $\mathrm{CC} / \mathrm{CG}+\mathrm{GG}$ & $10 / 68$ & $16 / 58$ & 0.15 \\
\hline
\end{tabular}

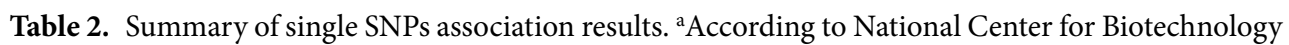
GRCh38.p12 assembly (June 21, 2019). ${ }^{b}$ Minor allele frequency (MAF) in CEU (Caucasian European) population. ${ }^{~}$ Fisher exact test, Bonferroni correction, significant if $\alpha \leq 0.005$. Italic font means $0.006 \leq \mathrm{P} \leq 0.009$.

\begin{tabular}{|c|c|c|c|c|c|c|}
\hline Genes & SNPs & Dataset & Haplotype & $\begin{array}{l}\text { Frequency of } \\
\text { cases }\end{array}$ & $\begin{array}{l}\text { Frequency of } \\
\text { controls }\end{array}$ & P value ${ }^{a}$ \\
\hline \multirow{4}{*}{ WNT3 } & \multirow{2}{*}{ rs111769/rs9890413 } & Pittsburgh & \multirow{2}{*}{ CG } & 0.24 & 0.10 & 0.00008 \\
\hline & & Combined & & 0.21 & 0.11 & 0.0002 \\
\hline & \multirow{2}{*}{ rs199498/rs111769//rs9890413 } & Pittsburgh & \multirow{2}{*}{ TCG } & 0.21 & 0.10 & 0.0005 \\
\hline & & Combined & & 0.18 & 0.11 & 0.002 \\
\hline \multirow{2}{*}{ WNT3//WNT9B } & \multirow{2}{*}{ rs9890413/rs2165846 } & Houston & AA & 0.21 & 0.35 & 0.007 \\
\hline & & Combined & GG & 0.21 & 0.13 & 0.004 \\
\hline \multirow{3}{*}{ WNT3/WNT9B } & \multirow{3}{*}{ rs111769/rs9890413/rs2165846 } & Pittsburgh & \multirow{2}{*}{ CGG } & 0.20 & 0.09 & 0.0002 \\
\hline & & Combined & & 0.17 & 0.08 & 0.0003 \\
\hline & & Houston & CAA & 0.13 & 0.25 & 0.007 \\
\hline \multirow{3}{*}{ WNT3/WNT9B } & \multirow{3}{*}{ rs199498/rs111769/rs9890413/rs2165846 } & Pittsburgh & \multirow{2}{*}{ TCGG } & 0.20 & 0.09 & 0.0009 \\
\hline & & Combined & & 0.15 & 0.08 & 0.002 \\
\hline & & Houston & TCAA & 0.12 & 0.24 & 0.009 \\
\hline WNT3A & rs752107/rs1745420 & Houston & CC & 0.23 & 0.39 & 0.003 \\
\hline
\end{tabular}

Table 3. Summary of haplotype analysis results. ${ }^{\text {BB }}$ Bonferroni correction, significant if $\alpha \leq 0.005$ (in bold). Italic font means $0.006 \leq \mathrm{P} \leq 0.009$.

protein functions. No transcription factor binding sites were identified for WNT3 rs9890413. For WNT3A rs1745420, results predicted that it may harbor eight conserved miRNA binding sites, suggesting potential effects on gene transcriptional activities (Supplementary Fig. S1). To further test for allele-specific differential transcriptional activities of WNT3A in vitro, a $3^{\prime}$ UTR reporter vector was used for plasmid construction and cloning of WNT3A rs $174520 \mathrm{C}$ and G alleles downstream of the luciferase reporter gene. Empty $3^{\prime}$ UTR vector was used as transfection control. Human embryonic kidney cells (HEK293T) cells were cultured following established protocols and transfected with each allele-specific construct. The results showed that the alternate allele $\mathrm{G}$ resulted in increased transcriptional activity (1.4-fold, $\mathrm{P}=0.03$ ) when compared to the ancestral allele $\mathrm{C}$ (Fig. 1 ).

Gene expression. We investigated the mRNA expression of the associated genes $W N T 3, W N T 3 A, W N T 5 A$, $W N T 9 B$ in AP lesion tissues using quantitative RT-PCR. Healthy periodontal ligament tissues were used as controls. The expression of WNT3, WNT3A and WNT5A mRNA was significantly higher in AP lesions when compared to controls, whereas no differences were observed for WNT9B expression. We also stratified AP lesions into active and inactive status, according to the methodology proposed by Menezes et al. ${ }^{13}$, and observed that expression of $W N T 3 A$ and $W N T 5 A$ was markedly higher in active lesions, while no differences were observed for WNT3 and WNT9B $(\mathrm{P}>0.05)$ (Fig. 2).

We also performed correlation analysis to assess the potential relationships between WNT gene expression levels with the expression of genes involved in bone healing (serpin family B member 1 (SERPINB1), TIMP metallopeptidase inhibitor 1 (TIMP1) and collagen type I alpha 1 chain (COL1A1). Expression of WNT3A and WNT5A was inversely correlated with the expression of SERPINB1 $\left(r^{2}=0.1759, \mathrm{P}<0.0001\right.$ and $r^{2}=0.1798, \mathrm{P}<0.0001$, respectively) and COL1A1 $\left(r^{2}=0.1774, \mathrm{P}<0.0001\right.$ and $r^{2}=0.0439, \mathrm{P}=0.0287$, respectively). Similarly, WNT3 expression was inversely correlated with TIMP1 expression $\left(r^{2}=0.0896, \mathrm{P}=0.0016\right)$. No significant correlation was observed for WNT9B (Fig. 3). 


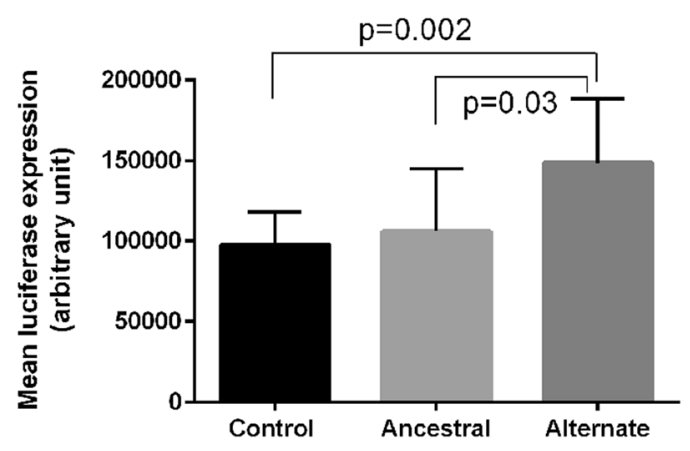

Figure 1. Results of luciferase reporter gene assays for WNT3A rs1745420. Allele-specific transcriptional activities are shown for ancestral (ANC) and (ALT) alleles.

\section{Discussion}

In this study, we assessed the potential association of WNT gene polymorphisms with AP risk in individuals with deep caries. We used a candidate gene association approach to investigate the association of SNPs in 11 WNT genes in 188 cases with AP and 230 control individuals without AP. Under the strict criteria of the Bonferroni correction $(\alpha=0.005)$, our findings revealed a trend for association between WNT3 (rs9890413) and WNT3A (rs1745420) with AP in the studied datasets.

AP has heterogeneous etiology and results from the dynamic interactions between pathogens and host defenses in the periapical tissues (i.e. those tissues surrounding the apex of a tooth), thus triggering a cascade of events with release of inflammatory mediators, local inflammation, and hard tissue breakdown characterizing an AP lesion ${ }^{2}$. Despite its widely accepted microbial origin, the development of AP has also been recently linked to the host immune respons $\mathrm{e}^{4,6}$. Clinical observations in individuals with deep caries vary; while in some individuals an AP lesion is detected quite readily in response to the microbial aggression, others are more resistant to developing an AP lesion in face of similar aggression. These findings are intriguing and suggest that genetic predisposition plays a role in the development of $\mathrm{AP}^{6}$.

Genetic polymorphisms have been reported to act as modifiers of diverse diseases and, as such, might influence the individual host response to the severity of the microbial challenge and/or the individual response to treatment of $\mathrm{AP}^{14}$. To date, a few studies in distinct populations and a recent meta-analysis support genetic predisposition as a risk factor towards increased AP susceptibility, with the most significant associations reported for polymorphisms in interleukin 1 beta $(I L 1 B)$, heat shock $70 \mathrm{kDa}$ protein 1-like (HSPA1L), MMP2 and MMP3 $3^{4-6,15}$.

The Wnt gene family consists of 19 members in humans, all of which are highly conserved genes that regulate gene expression, cell behavior, cell adhesion and cell polarity ${ }^{7}$. WNT proteins, following their binding to the FZD and/or LRP5/ 6 receptors, activate the canonical WNT/ $\beta$-catenin signaling pathway which in turn initiates a signaling transduction to several intracellular proteins to activate transcription of downstream genes ${ }^{7}$. Wnt $/ \beta$-catenin signaling proteins participate in multiple developmental events during embryogenesis and adult tissue homeostasis. In bone, active Wnt/ $\beta$-catenin signaling is critical, particularly for the differentiation of osteoblasts ${ }^{16}$. The activation of the canonical Wnt pathway in osteoblasts has been shown to upregulate OPG expression while downregulating RANKL expression, thereby suppressing bone resorption ${ }^{17}$. Moreover, defects or dysregulation of Wnt signaling are known to lead to various human skeletal diseases, such as osteogenesis imperfecta, osteoporosis, tetra-amelia (complete absence of all four limbs and other anomalies) and Robinow syndrome (short-limbed dwarfism and abnormalities in the head/face and external genitalia ${ }^{16}$. However, the role of WNT genes/proteins in normal periapical tissues and in AP is not completely elucidated.

Our association findings point towards a likely role for $W N T 3$ and $W N T 3 A$ towards increased AP risk and warrant additional studies using additional markers and other larger populations. The associated marker in WNT3 (rs9890413), albeit located upstream of the gene, was not predicted to harbor any functional effects on gene/protein function however it may be in linkage disequilibrium with a true causal variant ${ }^{18}$. Fine-mapping genotyping efforts around the WNT3 gene region may uncover the true causal variant.

Variations in WNT3 have also been associated with alterations in bone mineral density and implicated in osteoporosis ${ }^{19}$, and with nonsyndromic cleft lip/palate, a common craniofacial anomaly and a defect of bone development $^{20}$. Loss-of-function mutations in WNT3 are also known to cause tetra-amelia, an extremely rare autosomal recessive congenital disorder characterized by the absence of all four limbs, and multiple malformations in the body including the face, skull and reproductive organ $\mathrm{s}^{21}$. WNT3A is also known to be involved in craniofacial development ${ }^{16}$. Polymorphisms in $W N T 3 A$ were associated with oral squamous cell carcinoma; $W N T 3 A$ mRNA expression levels were also markedly higher in oral cancer tissues in comparison to normal mucosa ${ }^{22}$. Similar to WNT3, WNT3A rs 1745420 has also been associated with non-syndromic cleft lip/palate ${ }^{23}$. These findings strengthen the role of these genes in bone-related conditions and disorders, including AP. Further, WNT3A rs1745420 may have a regulatory role on gene expression/function, due to our observations of an increased transcriptional activity (1.4-fold) in the presence of the alternate allele $\mathrm{G}$ when compared to the ancestral allele C. These findings also suggest that the ancestral allele $\mathrm{C}$ may play a role in reducing translation and/or stability of the mRNA $^{24}$. rs1745420 is located next to several miRNA binding sites in the WNT3A gene (Supplementary Figs. S1 and S2) and may contribute to the modulation of gene expression post-transcriptionally ${ }^{25}$. 
WNT3

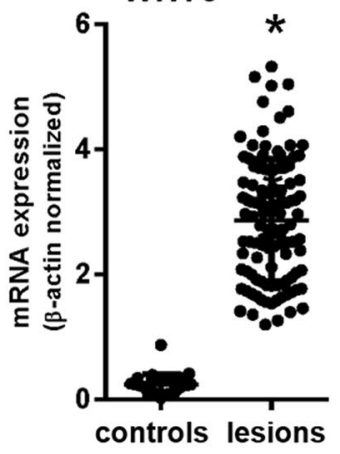

WNT3

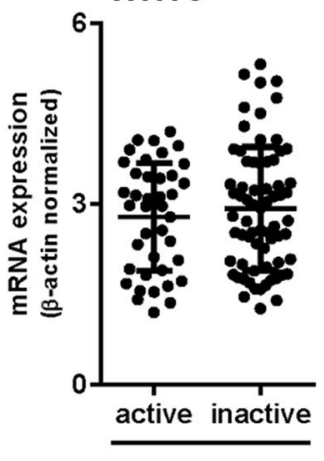

lesions

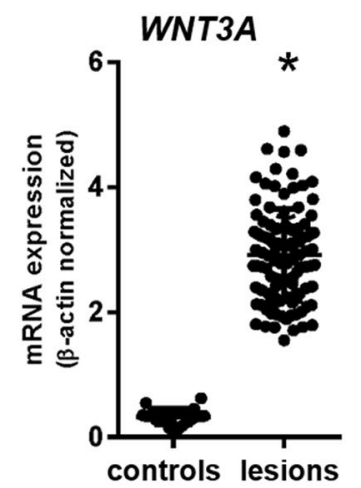

WNT3A

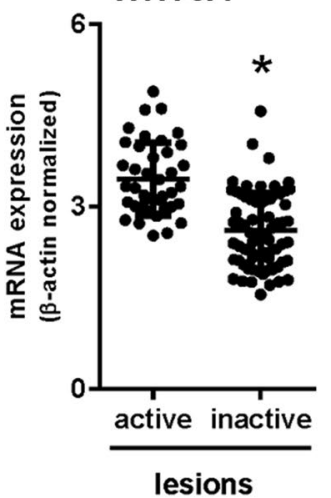

WNT5A
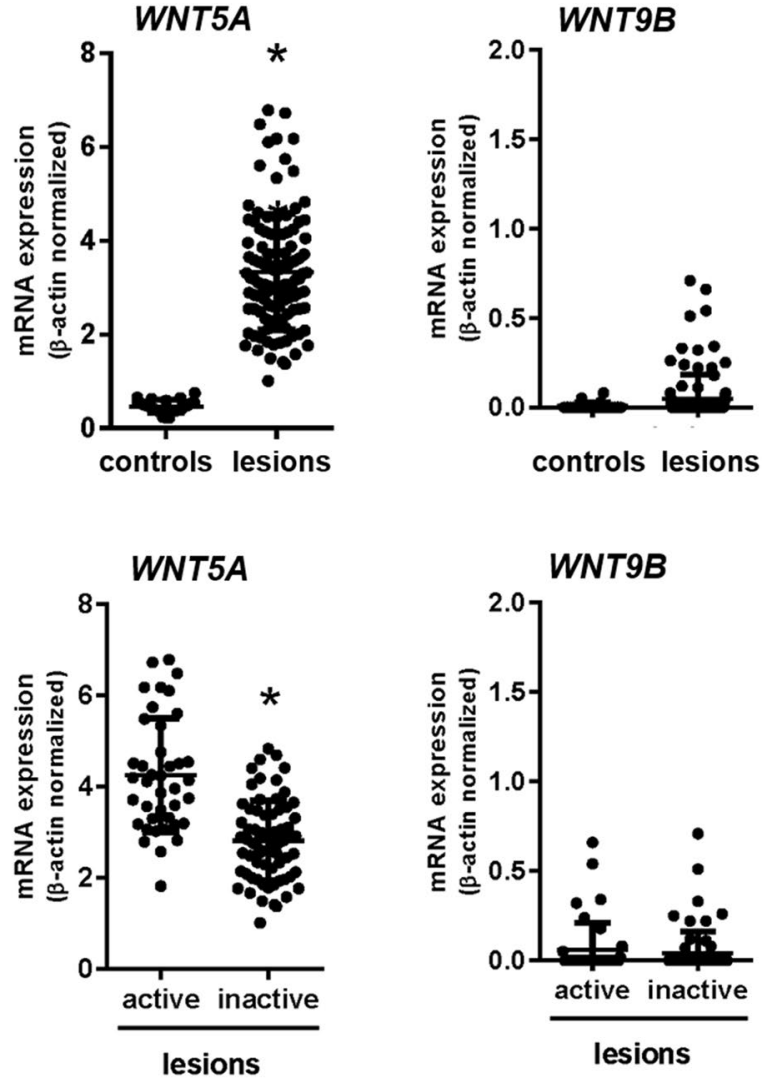

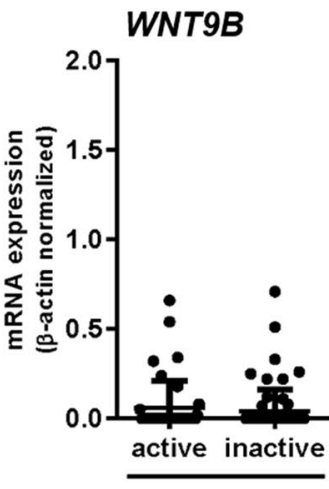

lesions

Figure 2. Expression of $W N T 3, W N T 3 A, W N T 5 A$ and $W N T 9 B$ mRNA in apical periodontitis (AP) and control tissues. Top, mRNA expression levels in AP lesions and controls. Bottom, mRNA expression levels in active and inactive AP lesions. *Indicates $\mathrm{P} \leq 0.05$.

Our findings of differential expression of WNT proteins in AP tissues further supports a role for these molecules in AP development. In the present study, WNT3, WNT3A and WNT5A expression was significantly higher in AP lesions when compared to control tissues. Interestingly, WNT3A and WNT5A expression was also upregulated in chronic periodontal disease tissues in comparison to healthy tissues ${ }^{26,27}$. Expression of WNT5A was upregulated in inflammatory systemic diseases, such as rheumatoid arthritis ${ }^{28}$, and also in AP tissues, in which the levels of expression of WNT5A increased with the severity of inflammation ${ }^{9}$. Further, WNT5A mRNA levels varied in a dose-depend manner when osteoblasts were exposed to Porphyromonas endodontalis lipopolysaccharide (LPS $)^{29}$. These observations of increased WNT3A and WNT5A expression in active lesions supports their involvement in AP lesion maintenance and/or progression.

We also assessed for potential correlations between the expression of the studied WNT genes with SERPINB1, $T I M P 1$, and COL1A1, known markers for wound healing and bone repair ${ }^{30}$. Significant inverse correlations were observed between the expression of WNT3A and WNT5A with SERPINB1, a potent inhibitor of neutrophil elastase secreted by neutrophils and involved in tissue destruction ${ }^{31}$. This suggests that increased expression of WNT3A and WNT5A may impact inhibition of neutrophil elastase thereby contributing to tissue destruction. Similarly, inverse correlations were found between the expression of WNT3A and WNT5A with COL1A1, which is an abundant and key molecule of the bone matrix ${ }^{32}$. WNT3 was also inversely correlated with TIMP1 expression. TIMP1 is a matrix metalloproteinase inhibitor with a critical role in bone remodeling and homeostasis; imbalances in TIMP gene expression may be related to increased or decreased bone resorption ${ }^{33}$. Interestingly, previous studies showed that SERPINB1, TIMP1 and COL1A1 were upregulated in AP lesions ${ }^{30,34}$. The expression of TIMP1 and COL1A1 was significantly higher in inactive AP lesions when compared to active lesions ${ }^{30}$. Similarly, TIMP1 was highly expressed in asymptomatic AP lesions, corroborating our findings ${ }^{35}$.

The identification of key molecules for AP has the potential to improve currently available therapies. Recently, in vitro studies have shown the potential therapeutic use of WNT/ $\beta$-catenin pathway molecules in AP treatment $^{8,11}$. The expression of Dickkopf-1 (Dkk1), an antagonist of the WNT signaling pathway, was detected in induced rat periapical lesions and implicated in the inflammatory response and associated bone resorption ${ }^{10}$. Dkk1 facilitates osteoclastogenesis by increasing expression of Rankl and decreasing the expression of Opg ${ }^{10}$. Further, the use of a Dkk1 inhibitor in a rat AP model restored bone loss, indicating that this might be a potential therapeutic target for $\mathrm{AP}^{11}$. Transient treatment with lithium chloride (LiCl), an inhibitor of GSK3 $\beta$, also resulted in bone formation and repair of induced AP lesions in rats, albeit continuous administration of the drug resulted in delayed bone repair ${ }^{8}$. 
WNT3 vs SERPINB1

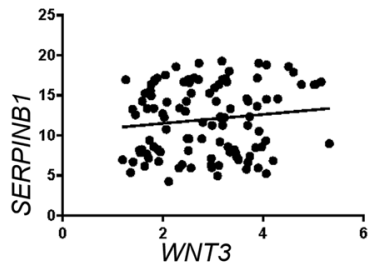

WNT3 vs TIMP1

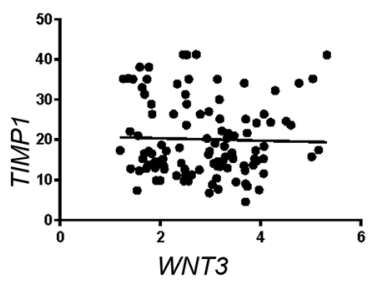

WNT3 vs COL1A1

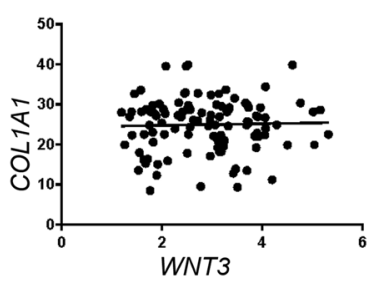

WNT3A vS SERPINB1

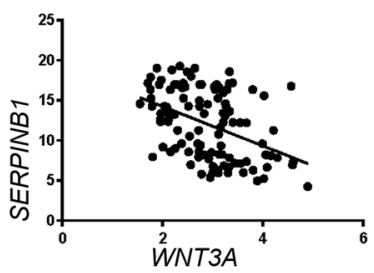

WNT3A vs TIMP1

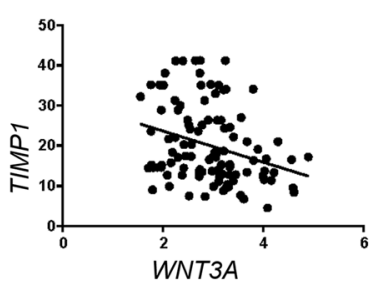

WNT3A vs COL1A1

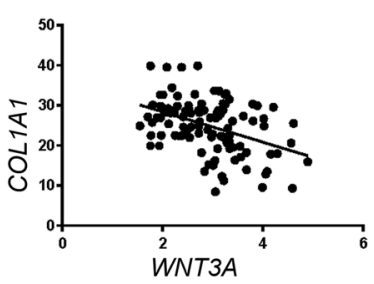

WNT5A vs SERPINB1

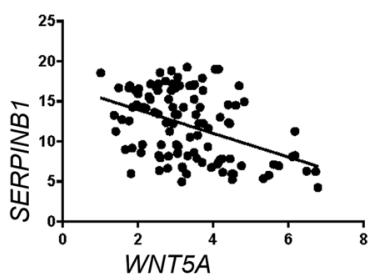

WNT5A vs TIMP1

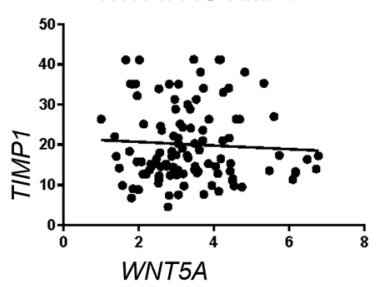

WNT5A vs COL1A1

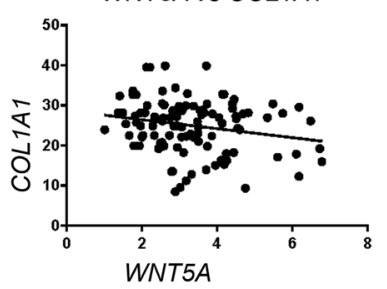

WNT9B vS SERPINB1

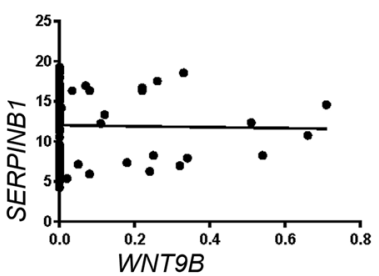

WNT9B vs TIMP1

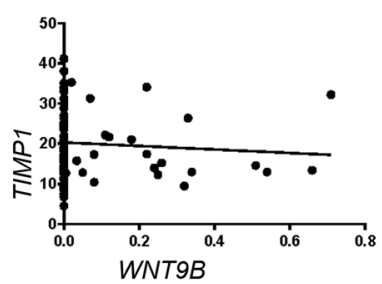

WNT9B vs COL1A1

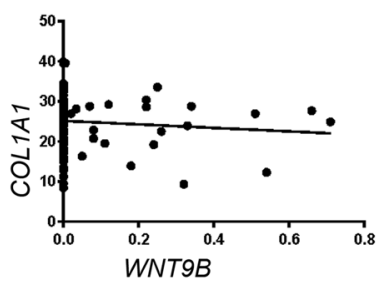

Figure 3. Correlation analysis between expression of WNT3, WNT3A, WNT5A and WNT9B with SERPINB1, TIMP1 and COL1A1 in AP lesions. *Indicates $\mathrm{P} \leq 0.05$.

Overall the findings of the present study provide additional insights into the modulatory role of Wnt genes during AP. Among the limitations of this study are the limited sample size and the focused approach on a given gene family. In contrast, a strength of the present study is the thorough phenotypic characterization of the study populations, which in turn increase confidence in the results obtained. One can argue that a hypothesis-free approach, like genome-wide association study or GWAS, would be a better approach for an association study, but so far, there is no proof that this approach might overcome issues of all genetic studies, such as epigenetic, cytogenetic and environmental cause ${ }^{36}$. Furthermore, it has been shown that expected associations might be neglected if a strict correction for multiple testing is applied when testing for several variants simultaneously ${ }^{37}$. Additional studies with different populations need to be done to confirm the association between WNT genes and $\mathrm{AP}$ development. Considering that $\mathrm{AP}$ is a multifactorial disease, involving endodontic microbiota and host response ${ }^{1}$, gene polymorphisms may be among the players influencing AP development.

In summary, AP derives jointly from host and microbial factors. Our study shows that WNT genes may have a modulatory role in AP development and suggest WNT3 and WNT3A as plausible contributor genes for AP. Dysregulation of contributor genes, as a result of genetic polymorphisms of the host, may influence destructive and/or remodeling events within the bone tissue. Identification of novel AP genes will elucidate the role of the host response in disease predisposition and pathogenesis and contribute towards improved treatment modalities.

\section{Methods}

Study populations and samples. This study followed the STROBE guidelines for human clinical studies. The Institutional Review Boards at the University of Pittsburgh (Project 0606091), University of São Paulo (Project 115/2009), Brazil, and the University of Texas Health Science Center at Houston (Project HSC-DB-12-0280) approved this study. The methods were carried out in accordance with the guidelines and regulations. Data extracted from electronic patient records and linked to DNA samples were provided in de-identified format. All individuals provided written informed consent agreeing to their participation in genetic studies. Three distinct datasets obtained at these institutions were used, as follows:

(a) Genetic association analysis: clinical/demographic history and saliva samples of individuals with and without AP were obtained at the University of Texas Health Science Center at Houston Graduate Endodontic Clinic and from the University of Pittsburgh Dental Registry and DNA Repository (DRDR) ${ }^{38}$. In brief, all individuals had to present one tooth showing a deep caries lesion (involving at least $2 / 3$ of the dentin depth). Individuals received standard-of-care clinical endodontic diagnostic testing (thermal and electric pulp vitality tests, palpation and percussion tests) for evaluation of pulpal and periapical status ${ }^{39}$. The presence of a periapical lesion was determined by evaluation of individual periapical radiographs, as a periapical rarefaction characterized radiographically by the disappearance of the periodontal ligament 
space and discontinuity of the lamina dura ${ }^{40}$. Only AP lesions $>3 \mathrm{~mm}$ were considered for this study. Based on clinical and radiographic findings, individuals were categorized as AP cases if in addition to deep caries, a pulpal diagnosis of asymptomatic irreversible pulpitis or pulp necrosis, and a periapical diagnosis of asymptomatic/ symptomatic AP or chronic apical abscess, and a periapical lesion $\geq 3 \mathrm{~mm}$ in diameter was found. Controls were individuals with deep caries, for which diagnostic testing confirmed a pulpal diagnosis of vital pulp tissues and/or irreversible pulpitis, and a periapical diagnosis of normal apical tissues (no AP lesion) or obvious widening of the periodontal ligament (PDL). The Pittsburgh dataset consisted of 109 AP cases and 155 controls (aged 20-60 years), whereas the Houston dataset had 79 cases and 75 controls (aged 18-65 years). Case and control groups were matched by age, sex, tobacco or alcohol usage.

(b) Gene expression analysis: discarded AP tissue samples were collected from individuals $(\mathrm{n}=110$, aged 19-61 years) referred to apical surgery or extraction after conventional root canal treatment had failed, at the University of Texas Health Science Center at Houston Dental Clinics and at the University of São Paulo (from 2009 to 2018). Upon collection, each sample was divided into 2 fragments of similar size and stored in $10 \%$ formalin or rinsed in PBS and immediately frozen. Samples stored in $10 \%$ formalin were submitted to histopathological analysis; frozen samples were used for gene expression analysis. All AP samples were histologically diagnosed as apical granulomas with or without epithelium (72\%) and radicular cysts (28\%). The clinical diagnosis included previously endodontically treated teeth $(100 \%)$ with asymptomatic apical periodontitis (43\%), symptomatic apical periodontitis (19\%), and chronic apical abscess (38\%). Healthy periodontal ligament tissues were collected from individuals referred extraction of premolars for orthodontic reasons $(n=26$, aged $19-24$ years) and used as control tissues.

Exclusion criteria for both analysis in this study included history of systemic conditions such as diabetes or other hormonal alterations related to exacerbated or uncontrolled inflammatory responses, and medical conditions requiring the use of systemic modifiers of bone metabolism or other assisted drug therapy (i.e., systemic antibiotics, anti-inflammatory, hormonal therapy) during the last 6 months before the study, preexisting conditions such as periodontal disease, and pregnant/ lactating women.

Selection of contributor genes. We selected eleven SNPs in/nearby WNT3, WNT3A, WNT5A, WNT8A, $W N T 9 B$ and WNT11 genes (Table 1). We prioritize the SNPs to be investigated in our study based on: (1) previous published reports, (2) their locations within their respective genes, (3) their potential functional consequences (i.e., located in regulatory regions such as promoter, $3^{\prime}$ UTR, exon, or exon/intron boundaries), and/or (4) considered tag-SNPs as surrogates for the linkage disequilibrium blocks surrounding the contributor gene ${ }^{12}$. We used information available at the NCBI dbSNP (http://www.ncbi.nlm.gov/SNP/) and 1000 Genomes (http:// www.1000genomes.org) databases to select polymorphisms.

Genotyping. Genomic DNA was extracted from saliva using established protocols. Genotypes were generated using Taqman chemistry ${ }^{41}$. Reactions were carried out in $5-\mu \mathrm{L}$ volumes in a ViiA7 Sequence Detection System (Applied Biosystems, Foster City, CA). In order to ensure quality control of genotyping reactions, a non-template control (water instead of DNA) was used as negative control and a DNA sample of known genotype as positive control. Genotype calls were automated using the TaqMan Genotyper Software (Applied Biosystems), and only automatic calls with a $>95 \%$ call rate were considered.

In Silico bioinformatic analysis of SNV function. We used the FuncPred tool on the SNPinfo web server (https://snpinfo.niehs.nih.gov/snpinfo/snpfunc.html) and TargetScan (http://www.targetscan.org) for in silico prediction of SNV function for the associated WNT3 rs9890413 and WNT3A rs1745420.

Luciferase reporter assay. A reporter construct with 51 base pairs containing the WNT3A rs $174520 \mathrm{C} / \mathrm{G}$ variant was cloned downstream of the luciferase reporter gene of a $3^{\prime}$ UTR Reporter Vector (Active Motif, Carlsbad, CA), which contained RenSP, an optimized Renilla luminescent reporter gene. Cloning and sequence validation were obtained using Switchgear genomics custom cloning service (Active Motif). Amplicons sequences were: CACACCTGGCCTGCAAGGGGACCTTCGGCTCTCCACCCAGATGGCCCCCTG (allele C), and CACACCTGGCCTGCAAGGGGACCTTGGGCTCTCCACCCAGATGGCCCCCTG (allele G). Empty 3' UTR vector (S890005, Active Motif) was used as positive control for transfection.

HEK293T cells (ATCC CRL-11268, ATCC, Manassas, VA) were cultured in Dulbecco's Modified Eagle's Medium supplemented with $10 \%$ fetal bovine serum and $1 \%$ penicillin/streptomycin at $37^{\circ} \mathrm{C}$ in $5 \% \mathrm{CO}_{2}$ atmosphere. Cells were seeded at a density of 15,000 cells/well in 96-well plates (Corning, Kennebunk, ME), cultured to $80 \%$ confluence, transfected with $50 \mathrm{ng}$ of each construct using FuGENE HD (Promega, Madison, WI) in OptiMEM medium (Life Technologies, Grand Island, NY) and cultured for 48 hours. Cells were lysed at $-80^{\circ} \mathrm{C}$ overnight, thawed and incubated with $100 \mathrm{uL} /$ well of LightSwitch reagent (Active Motif) for 30 minutes at room temperature. Luciferase activity was measured in a Tecan Infinite 200 Pro reader (Tecan, San Jose, CA) and normalized to Renilla luciferase activity levels. Three independent experiments were conducted in sextuplicates for each construct. Luciferase activity was recorded as arbitrary units of fluoresce (AU). Data is presented as mean \pm standard deviation of three independent experiments.

Gene expression assays. Total RNA was extracted using RNAeasy kit (Qiagen, Valencia, CA). RNA quality was analyzed using 2100 Bioanalyzer (Agilent Technologies, Santa Clara, CA), and complementary DNA was synthesized using $3 \mu \mathrm{g}$ RNA/sample as a template in a reverse-transcription reaction (QuantiTectRT Kit, Qiagen). AP tissues were first categorized into active/progressive $(\mathrm{n}=40$; RANKL $>$ OPG) or inactive/stable $(\mathrm{n}=70 ; \mathrm{RANKL} \leq \mathrm{OPG})$ based on RANKL/OPG expression ratios as previously described ${ }^{13}$. Next, we evaluated 
the mRNA levels of WNT3, WNT3A, WNT5A, WNT9B, SERPINB1, TIMP1 and COL1A1 in the active and inactive AP lesions using TaqMan gene expression assays (Invitrogen, Carlsbad, CA) in a ViiA7 Real Time PCR instrument (Life Technologies, Carlsbad, CA). Experiments were run in triplicates and repeated twice.

Statistical analysis. Power calculations were performed using GAS Power Calculator (http://csg.sph.umich. edu/abecasis/cats/gas_power_calculator/index.html) and indicate that the proposed sample size will provide $\sim 97 \%$ statistical power to detect an association with an alpha of 0.05 , under a multiplicative disease model, prevalence and disease allele frequency of $25 \%$ (Supplementary Fig. S2).

We tested for deviation from Hardy-Weinberg equilibrium (HWE) using PLINK v.1.05 software ${ }^{42}$. Allele and genotype frequencies for each SNP in cases and controls were compared using Fisher Exact tests as implemented in PLINK. Haplotype association analyses were performed for SNPs in chromosome 1 (WNT3A) and 17 (WNT3 and WNT9B) using 'haplotype-based case-control test' function. Bonferroni correction was used to adjust for multiple testing (0.05/11 SNPs) and $\alpha \leq 0.005$ indicated statistically significant differences between groups.

For the luciferase assay, differences between groups were tested using Kruskal-Wallis and post hoc Dunn's test, as implemented in GraphPad Prism 7 (GraphPad, La Jolla, CA).

Expression levels of target genes relative to the expression of housekeeping genes (GAPDH and $\beta$-actin) in each sample were analyzed using the $2{ }^{\Delta \Delta} \mathrm{Ct}$ method ${ }^{43}$. Statistical differences between groups were assessed using Mann-Whitney test and linear regression. Analyses were performed in GraphPad Prism 7. P $\leq 0.05$ indicated statistically significant differences between groups.

\section{Data availability}

Individual level data for any of the experiments described in the study may be available upon request.

Received: 7 August 2019; Accepted: 11 November 2019;

Published online: 12 December 2019

\section{References}

1. Nair, P. N. Pathogenesis of apical periodontitis and the causes of endodontic failures. Crit Rev Oral Biol Med 15, 348-381 (2004).

2. Roças, I. N. \& Siqueira, J. F. Jr. Root canal microbiota of teeth with chronic apical periodontitis. J Clin Microbiol 46, 3599-3606 (2008).

3. Takahama, A. Jr. et al. Association between bacteria occurring in the apical canal system and expression of bone-resorbing mediators and matrix metalloproteinases in apical periodontitis. Int Endod J 51, 738-746 (2018).

4. Dill, A. et al. Analysis of multiple cytokine polymorphisms in individuals with untreated deep carious lesions reveals IL1B (rs1143643) as a susceptibility factor for periapical lesion development. J Endod 41, 197-200 (2015).

5. Maheshwari, K. et al. Heat Shock 70 Protein Genes and Genetic Susceptibility to Apical Periodontitis. J Endod 42, 1467-1471 (2016).

6. Menezes-Silva, R., Khaliq, S., Deeley, K., Letra, A. \& Vieira, A. R. Genetic susceptibility to periapical disease: conditional contribution of MMP2 and MMP3 genes to the development of periapical lesions and healing response. J Endod 38, 604-607 (2012).

7. van Amerongen, R. \& Nusse, R. Towards an integrated view of Wnt signaling in development. Development 136, 3205-3214 (2009).

8. Tang, Y. et al. Modulation of Wnt/beta-catenin signaling attenuates periapical bone lesions. J Dent Res 93, 175-182 (2014).

9. Xu, L. Y. et al. Expression of Wnt5a in chronic apical periodontitis and its clinical significance. Shanghai Kou Qiang Yi Xue 24, 470-474 (2015).

10. Zhang, R., Huang, S., Wang, L. \& Peng, B. Histochemical localization of Dickkopf-1 in induced rat periapical lesions. J Endod 40 , 1394-1399 (2014).

11. Tan, X. et al. Dickkopf-1 may regulate bone coupling by attenuating wnt/beta-catenin signaling in chronic apical periodontitis. Arch Oral Biol 86, 94-100 (2018).

12. Carlson, C. S. et al. Selecting a maximally informative set of single-nucleotide polymorphisms for association analyses using linkage disequilibrium. Am J Hum Genet 74, 106-120 (2004).

13. Menezes, R. et al. Differential patterns of receptor activator of nuclear factor kappa B ligand/osteoprotegerin expression in human periapical granulomas: possible association with progressive or stable nature of the lesions. J Endod 34, 932-938 (2008).

14. Mehrazarin, S., Alshaikh, A. \& Kang, M. K. Molecular Mechanisms of Apical Periodontitis: Emerging Role of Epigenetic Regulators. Dent Clin North Am 61, 17-35 (2017).

15. Aminoshariae, A. \& Kulild, J. C. Association of Functional Gene Polymorphism with Apical Periodontitis. J Endod 41, 999-1007 (2015).

16. Wang, Y. et al. Wnt and the Wnt signaling pathway in bone development and disease. Front Biosci (Landmark Ed) 19, 379-407 (2014).

17. Maeda, K., Takahashi, N. \& Kobayashi, Y. Roles of Wnt signals in bone resorption during physiological and pathological states. J Mol Med (Berl) 91, 15-23 (2013).

18. Eckert, S. et al. Variants on chromosome 4q21 near PKD2 and SIBLINGs are associated with dental caries. J Hum Genet 62, 491-496 (2017).

19. Mo, X. B. et al. Gene-based association analysis identified novel genes associated with bone mineral density. PLoS One 10, e0121811 (2015).

20. Menezes, R. et al. Studies with Wnt genes and nonsyndromic cleft lip and palate. Birth Defects Res A Clin Mol Teratol 88, 995-1000 (2010).

21. Niemann, S. et al. Homozygous WNT3 mutation causes tetra-amelia in a large consanguineous family. Am J Hum Genet 74, 558-563 (2004).

22. Andrade Filho, P. A. et al. Insights from studies with oral cleft genes suggest associations between WNT-pathway genes and risk of oral cancer. J Dent Res 90, 740-746 (2011).

23. Chiquet, B. T. et al. Variation in WNT genes is associated with non-syndromic cleft lip with or without cleft palate. Hum Mol Genet 17, 2212-2218 (2008).

24. Song, C., Chen, L. Z., Zhang, R. H., Yu, X. J. \& Zeng, Y. X. Functional variant in the $3^{\prime}$-untranslated region of Toll-like receptor 4 is associated with nasopharyngeal carcinoma risk. Cancer Biol Ther 5, 1285-1291 (2006).

25. Han, S. et al. The effects of alternative splicing on miRNA binding sites in bladder cancer. PLoS One 13, e0190708 (2018).

26. Nanbara, H. et al. Modulation of Wnt5a expression by periodontopathic bacteria. PLoS One 7, e34434 (2012).

27. Liu, X., Zhang, Z., Pan, S., Shang, S. \& Li, C. Interaction between the Wnt/beta-catenin signaling pathway and the EMMPRIN/ MMP-2, 9 route in periodontitis. J Periodontal Res (2018).

28. Kim, J. et al. Wnt5a induces endothelial inflammation via beta-catenin-independent signaling. J Immunol 185, 1274-1282 (2010). 
29. Yu, Y. et al. TRIB3 mediates the expression of Wnt5a and activation of nuclear factor-kappaB in Porphyromonas endodontalis lipopolysaccharide-treated osteoblasts. Mol Oral Microbiol 30 (2015).

30. Garlet, G. P. et al. Expression analysis of wound healing genes in human periapical granulomas of progressive and stable nature. J Endod 38, 185-190 (2012).

31. Uchiyama, K. et al. Serpin B1 protects colonic epithelial cell via blockage of neutrophil elastase activity and its expression is enhanced in patients with ulcerative colitis. Am J Physiol Gastrointest Liver Physiol 302, G1163-1170 (2012).

32. Viguet-Carrin, S., Garnero, P. \& Delmas, P. D. The role of collagen in bone strength. Osteoporos Int 17, 319-336 (2006).

33. Sobue, T. et al. Tissue inhibitor of metalloproteinases 1 and 2 directly stimulate the bone-resorbing activity of isolated mature osteoclasts. J Bone Miner Res 16, 2205-2214 (2001).

34. Cavalla, F. et al. Proteomic Profiling and Differential Messenger RNA Expression Correlate HSP27 and Serpin Family B Member 1 to Apical Periodontitis Outcomes. J Endod 43, 1486-1493 (2017).

35. Letra, A. et al. MMP-7 and TIMP-1, new targets in predicting poor wound healing in apical periodontitis. J Endod 39, 1141-1146 (2013).

36. Vieira, A. R. Hypothesis-driven versus hypothesis-free approaches to the identification of genes for cleft lip and palate. Arch Oral Biol 92, 88-89 (2018).

37. Vieira, A. R., McHenry, T. G., Daack-Hirsch, S., Murray, J. C. \& Marazita, M. L. Candidate gene/loci studies in cleft lip/palate and dental anomalies finds novel susceptibility genes for clefts. Genet Med 10, 668-674 (2008).

38. Vieira, A. R., Hilands, K. M. \& Braun, T. W. Saving more teeth-a case for personalized care. J Pers Med 5, 30-35 (2015).

39. Glickman, G. N. AAE Consensus Conference on Diagnostic Terminology: background and perspectives. J Endod 35, 1619-1620 (2009).

40. Huumonen, S. \& Ørstavik, D. Radiological aspects of apical periodontitis. Endodontic Topics 1, 3-25 (2002).

41. Ranade, K. et al. High-throughput genotyping with single nucleotide polymorphisms. Genome Res 11, 1262-1268 (2001).

42. Purcell, S. et al. PLINK: a tool set for whole-genome association and population-based linkage analyses. Am J Hum Genet 81, 559-575 (2007).

43. Livak, K. J. \& Schmittgen, T. D. Analysis of relative gene expression data using real-time quantitative PCR and the 2(-Delta Delta C(T)) Method. Methods 25, 402-408 (2001).

\section{Acknowledgements}

The authors deny any conflicts of interest. The authors are thankful to the study participants. This work was supported in part by NIH/NIDCR RO1-DE18914 (to ARV), FAPESP grants 15/24637-3 (to G.P.G.) and 14/03276-0 (to F.C.), Advanced Human Resources Training program CONICYT (Chile) (to F.C.), and the American Association of Endodontists Foundation Research Grants (to A.L. and R.M.S.).

\section{Author contributions}

L.C.S. contributed to data acquisition, analyses and interpretation, and drafted the manuscript. F.C., L.M. and A.R.V., contributed to data acquisition, analyses and interpretation. A.L., R.M.S. and G.P.G. contributed to study design, data analyses and interpretation. A.L. contributed to study conception, design, data analysis and interpretation. L.C.S. and A.L. drafted the manuscript. All authors critically reviewed and approved the manuscript.

\section{Competing interests}

The authors declare no competing interests.

\section{Additional information}

Supplementary information is available for this paper at https://doi.org/10.1038/s41598-019-55293-6.

Correspondence and requests for materials should be addressed to A.L.

Reprints and permissions information is available at www.nature.com/reprints.

Publisher's note Springer Nature remains neutral with regard to jurisdictional claims in published maps and institutional affiliations.

(c) (i) Open Access This article is licensed under a Creative Commons Attribution 4.0 International License, which permits use, sharing, adaptation, distribution and reproduction in any medium or format, as long as you give appropriate credit to the original author(s) and the source, provide a link to the Creative Commons license, and indicate if changes were made. The images or other third party material in this article are included in the article's Creative Commons license, unless indicated otherwise in a credit line to the material. If material is not included in the article's Creative Commons license and your intended use is not permitted by statutory regulation or exceeds the permitted use, you will need to obtain permission directly from the copyright holder. To view a copy of this license, visit http://creativecommons.org/licenses/by/4.0/.

(c) The Author(s) 2019 